\title{
Adaptive Hybrid Mobile Agent Protocol for Wireless Multihop Internet Access
}

\author{
${ }^{1}$ A. Velmurugan and ${ }^{2} \mathrm{R}$. Rajaram \\ ${ }^{1}$ Department of Electronics Engineering, Madras Institute of Technology, Chennai, India-600 044 \\ ${ }^{2}$ Department of Information Technology, Thiagarajar College of Engineering, Madurai, India
}

\begin{abstract}
Internet-based Mobile Ad Hoc Networking (MANET) is an emerging technology that supports self-organizing mobile networking infrastructures. This is expected to be of great use in commercial applications for the next generation Internet users. A number of technical challenges are faced today due to the heterogeneous, dynamic nature of this hybrid MANET. A new hybrid routing scheme AODV_ALMA is proposed, which act simultaneously combining mobile agents to find path to the gateway to establish connection with Internet host and on-demand distance vector approach to find path in local MANET is one of the unique solution. An adaptive gateway discovery mechanism based on mobile agents making use of pheromone value, pheromone decay time and balance index is used to estimate the path and next hop to the gateway. The mobile nodes automatically configure the address using mobile agents first selecting the gateway and then using the gateway prefix address. The mobile agents are also used to track changes in topology enabling high network connectivity with reduced delay in packet transmission to Internet. The performance tradeoffs and limitations with existing solutions for various mobility conditions are evaluated using simulation.
\end{abstract}

Key words: Hybrid MANET, mobile agents, AODV_ALMA, adaptive gateway discovery

\section{INTRODUCTION}

A Mobile Ad Hoc Network (MANET) is an autonomous network that can be formed without of any established infrastructure. It consists of mobile nodes equipped with a wireless interface that are free to move and communicate. The mobile nodes act as host and routers, which has routing capabilities that create multihop paths connecting nodes which cannot directly communicate. However, the idea of facilitating the integration of MANETs and fixed IP networks has gained a lot of momentum within the research community of late ${ }^{[1]}$. In the near future the mobile nodes are expected to form dynamic hybrid MANET which provide service information for ubiquitous access. The hybrid MANET can be deployed quickly and easily for potential applications like conference meeting, wireless classrooms, vehicular operations in a highway, ubiquitous information processing in train and airport.

Researchers are now making use of many relatively simple biological agents like Ants and are developing a variety of different organized behaviors at the system level. Because of the robustness and efficiency they have recently become a source of inspiration for the design of routing algorithms called Ant algorithm for dynamic networks. And also Ant Colony Optimization (ACO) meta heuristic scheme using Swarm Intelligence has been developed based on the ant societies.

The Ant like Mobile agents (ALMA) are simple packets that explore the network and collect data to be used for routing ${ }^{[2,3]}$.

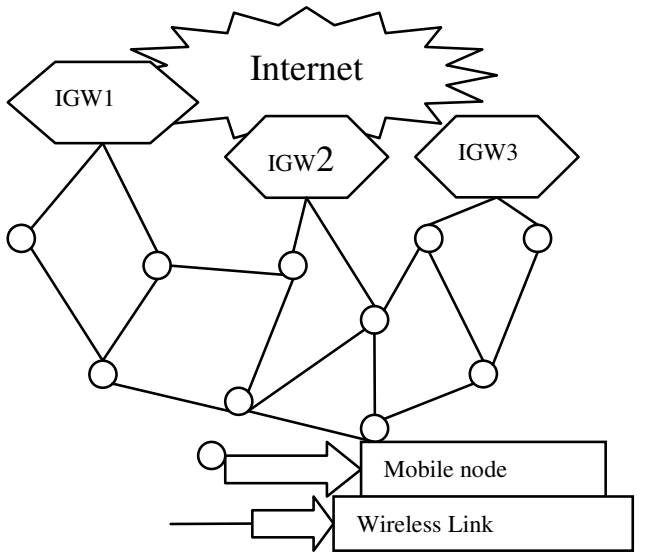

Fig. 1: Mobile ad hoc network connectivity with Internet

They communicate with one another indirectly to exchange routing information by writing and reading data to /from routing tables as ants exchange food information with each other. By using mobile agent exploration, we put the intelligence across the network and make the routing distributed with high connectivity. Furthermore, in mobile agent based routing protocols the overhead is proportional to the number of agents. This in return reduces the overhead compared with traditional routing protocols that use frequent updates and reactive updates.

This study addresses to solve the technical challenges of hybrid MANET shown in Fig. 1. 
The main technical challenges faced today in this hybrid MANET are Internet gateway discovery, address auto-configuration and reaching the destination. An ant algorithm is used as an adaptive reactive gateway discovery approach integrated with address autoconfiguration. The new scheme developed is named as AODV_ALMA.

\section{RELATED EXISTING PROPOSALS}

The first proposals by Broch et al. ${ }^{[4]}$ are based on integration of Mobile IP and MANET employing a source routing protocol. MIPMANET ${ }^{[5]}$ followed a similar approach based on AODV, but it only works with Mobile IPv4, because it requires Foreign Agents (FAs). In addition, Ammari et al. ${ }^{[6]}$ analyzed the performance of mobile gateways in a MANET based on the DSDV protocol.

$\mathrm{In}^{[7]}$ the authors propose a scheme in which advertisements are only propagated up to a certain number of hops. Nodes located out of that scope will reactively find the gateways when needed. However, the performance of this approach depends on the TimeTo-Live (TTL) value which is set on particular scenario and network conditions under consideration. $\operatorname{In}^{[8]}$ the authors propose a more sophisticated approach in which advertisements are sent out only when changes in topology are detected. However, they rely on a sourcebased routing protocol, which limits the applicability of their approach to this particular type of routing protocol. $\operatorname{In}^{[9]}$ an adaptive gateway discovery mechanism that outperforms existing hybrid approaches is proposed. The key is that the TTL value for proactive gateway advertisements is adjusted dynamically to network conditions. Those mobile nodes out of that scope reactively find the gateways. The proposals available till date focused only on gateway discovery and are not a complete solution for inter-working with fixed IP networks.

Some complete proposals which integrate both auto-configuration and gateway discovery namely Wakikawa $^{[10]}$, Jelger ${ }^{[11]}$ and Singh et $a l^{[12]}$. In Wakikawa mobile nodes discover the gateway either reactively or proactively to configure the IP address automatically. In the proactive approach, gateway periodically floods the gateway advertisement (GWADV) messages up to certain number of hops in the MANET. In the reactive approach, the gateway solicitation (GWSOL) message is flooded from the mobile node and unicast gateway advertisement (GWADV) returns from the gateway to the mobile node. The gateway advertisement message contains the global IPv6 address of the gateway, the network prefix advertised by the gateway, the prefix length and the lifetime associated with this information.

In Jelger et al. ${ }^{[11]}$ proposal, proactive approach is used in which the gateways advertise themselves by periodically flooding gateway information (GW_INFO)
Table 1: Routing table in mobile nodes

Destination address

Next Hop address

Number of Hops

Sequence number for the destination

Active neighbors for this route

Expiration time for the route table entry

Pheromone value

Pheromone decay time

Balance index

Table 2: Routing table in gateways

Source address

Next Hop address

Number of Hops

Sequence number for the source

Active neighbors for this route

Expiration time for the route table entry

Pheromone value

Pheromone decay time

Balance index

messages. The flooding of these messages is based on the idea of prefix continuity. A mobile node selects its best path toward the gateway using one of the metrics distance or stability and or delay from all the gateway information messages received. By selecting the gateway information messages, the mobile node configures an IPv6 address based on the selected prefix. This approach does not require the use of routing headers.

In Singh et al. ${ }^{[12]}$ proposal, gateways act as those nodes that are not one hop away from the access router. Access routers are expected to be equipped with a wireless interface. The first node becoming a gateway is called the default gateway and it is responsible for sending out periodic gateway advertisements. The other gateways for a given router are called Candidate gateways. A Candidate gateway becomes a default gateway when it stops receiving gateway advertisements from the default gateway for some time. It defines gateway selection based on bandwidth balancing, but unfortunately bandwidth parameters and their use not described in the current version of the specification.

\section{PROPOSED PROTOCOL DESCRIPTION AODV_ALMA}

In hybrid MANET the network connectivity of mobile nodes with gateway and mobile nodes with other active nodes is uncertain due to the dynamic network topology. The mobility of mobile nodes will cause more delay in finding the route to the destination either in the Internet or in the MANET.

The new proposal handles this situation by using hybrid mechanism, pure on-demand distance vector mechanism i.e., AODV used in finding the path within MANET and ACO mechanism i.e., ALMA (Ant like Mobile Agents) used in finding the path to the gateway. The hybrid mechanism operates simultaneously when a route to the destination is needed by the source. The routing table structure in mobile node is dynamically 
updated on demand is shown in Table 1. The gateway contains two routing tables, one to update information from MANET and another one to update the information from Internet is shown in Table 2.

The data packets are forwarded either to the gateway or to the mobile node in MANET using the routing table entries available reactively. The data packets to the fixed destination are forwarded to the gateway by using the entries such as number of hops, next hop, pheromone values and pheromone decay time and balance index. The data packets to the destination in MANET are forwarded by using entries such as number of hops, next hop and Active neighbors for this route and Expiration time. The data packets transmitted use IPv6 header information both for gateway and in MANET.

The main reason for using ACO in gateway discovery is to set adaptive parameters based on current topology of the hybrid MANET. The other reason is to take decision about selecting the next gateway and the next hop to the gateway which is based on the pheromone concentration on the current node provided for each possible link. In this proposal AODV_ALMA, the ACO principle is a unifying framework with ondemand distance vector approach which is used to evaluate the network connectivity and End-to-End delay.

Gateway discovery in AODV_ALMA: The proposed algorithm use AODV_ALMA (Ant-like mobile agents) to discover Internet gateway (IGW) and maintain paths in MANET to the fixed Internet based on dynamic topology. Initially when the mobile nodes are to find a route to the destination it broadcasts the available address using the Forward Routing Mobile Agent (FRMA) used to find a path connecting source and IGW. The Route Request Message (RREQ) used to find a path in the MANET. The FRMA packet contains the IPv6 address for the group of all gateways in the hybridMANETi.e., ALL_MANET_GW_MULTICAST address. The FRMA packet is rebroadcast in the MANET until it discovers the gateway. The FRMA keeps a list of the nodes $[1,2 \ldots, \mathrm{n}]$ it has visited. In the forward direction the FRMA packet updates the routing table of active nodes with probability value for the pheromones. The gateway finding the FRMA packet updates the routing table of its own by the source address in the FRMA packet and also the pheromone value available in the packet. The pheromone value present in the FRMA packet is bound to decay with time and number of hops traveled. If Larger the value of pheromone, then shorter the time taken to reach the gateway, but smaller the value of pheromone longer the time to reach the gateway. After reaching the gateway FRMA packet is converted to Backward Routing Mobile Agent (BRMA) packet which has got higher priority over FRMA packet.

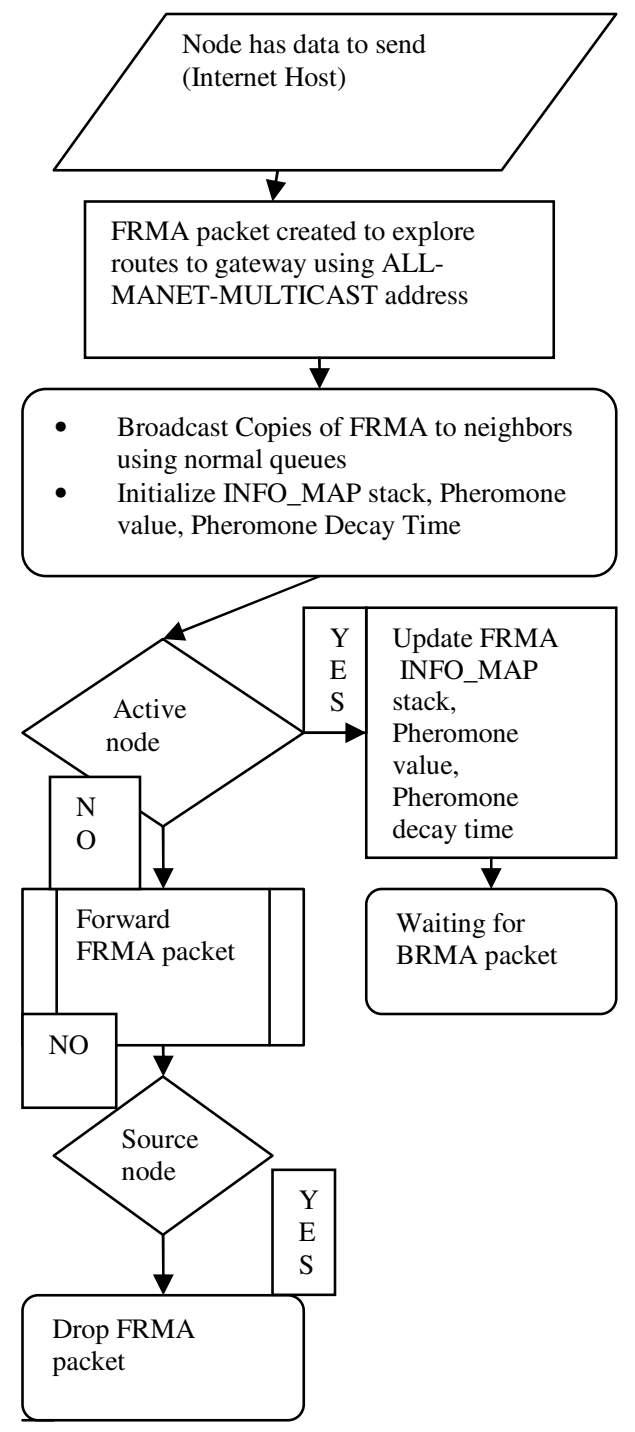

Fig. 2: Process in mobile source node using AODV_ALMA

The BRMA packet contains the global IPv6 address of the gateway, the network prefix of the gateway, the prefix length, lifetime associated with this information and the balance index in a unicast fashion to the source node. The BRMA packet updates the reverse path to the source with the available information in the active nodes. After reaching the source the BRMA packet updates the routing table. When a data packet is to be sent to the Internet host, it is forwarded based on the information available in the source node updated by BRMA packets i.e., pheromone value, pheromone decay time and balance index. The adaptive gateway discovery scheme is used to select the gateway based on parameters updated by BRMA packet. The source mobile node selects the gateway according to the pheromone value, pheromone decay time and balance index. The source node which receives more than one BRMA packet configures its IP address after adaptively selecting the gateway. 
The active nodes maintain the adaptive parameters, reaching a threshold value generates a new FRMA packet with source address available in the routing table. The generated FRMA packet updates the route to the gateway with a new active node to be selected for forwarding the data packets. The FRMA and BRMA packets explore and quickly reinforce newly discovered paths to the gateway in the hybrid MANET. Further, they ensure that previously discovered paths do not get saturated. This configuration is used to discover the topology of hybrid MANET dynamically, which increases the connectivity of the network leading to less delay in packet transmission. The flowchart shows the process in a mobile node in Fig. 2.

Analytical model: In the analytical evaluation of AODV_ALMA, the Fixed Radius Model is used as the topology model for hybrid MANET. Let $\mathfrak{R}_{\text {be the }}$ node's communication range. In the first case, every mobile node connects to the nearest gateway. To prevent mobile nodes from being isolated from the network, gateways must therefore support multihop connection in hybrid MANET. In the second case, the density and radius must be large enough to keep the proportion of nodes which do not belong to the geographical area is small enough. The Ant like Mobile Agents (ALMA) can be used to find the path between a source node s and the gateway $g$ on the connected graph $G=(V, E)$ with $n=|V|$ nodes. The path length is given by the number of nodes on the path. Each edge $e(i, j) \in E$ of the graph connecting the nodes on the path, $v_{i}$ and $v_{j}$ has a pheromone value $\varphi_{i, j}$ which is modified by the ALMA packets when they visit the active node.

In order to forward a packet towards its gateway g, the forwarding equation is used to determine the next hop neighbor. This formula maps the gateway g pheromone on each outgoing link $\mathrm{j}$ at node $\mathrm{i}, p_{i, j, g}$, to the pheromone that link will be used to forward the packet to the gateway $\varphi_{i, j, g}$. The specific next hop neighbor is chosen randomly according to this distribution. The forwarding equation is shown in equation (1)

$p_{i, j, g}=\frac{\left(\varphi_{i, j, g}+K\right)^{F}}{\sum_{j=1}^{N_{i}}\left(\varphi_{i, j, g}+K\right)^{F}} \quad$ if $j \in N_{i}$

$0 \quad$ if $j \notin N_{i}$

$N_{i}$ is the number of neighbors of node $\mathrm{j}$. The constants $\mathrm{F}$ and $\mathrm{K}$ are used to tune the routing behavior of AODV_ALMA. The pheromone threshold, $\mathrm{K}$, determines the sensitivity of the pheromone calculations to small amounts of pheromone. If $K \geq 0$ is large, then large amounts of pheromone will have to be present before an appreciable effect will be seen in the routing probability. Similarly, the pheromone sensitivity, $F \geq 0$, may be used to modulate the differences between pheromone amounts. For example, F $>1$ will accentuate differences between links, while $\mathrm{F}<1$ will deemphasize them. $\mathrm{F}=1$ yields a simple normalization.

The transition probabilities $p_{i, j, g}$ of a node $v_{i}$ fulfill the constraint:

$$
\sum_{j \in N_{i}} p_{i, j, g}=1, i \in[1, N]
$$

During the route finding process to the gateway in AODV_ALMA, FRMA and BRMA packets deposit pheromone on the edges. The pheromone concentration is varying in a constant amount $\Delta \varphi$. The change in the amount of pheromone at the edges $e\left(v_{i}, v_{j}\right)$ when moving from node $v_{i}$ to node $v_{j}$ is as follows:

$$
\varphi_{i, j, g}:=\varphi_{i, j, g}+\Delta \varphi
$$

The pheromone value $\varphi_{i, j, g}$ can be used to compute the probability $p_{i, j, g}$ of the node $v_{j}$ as the next hop to gateway $\mathrm{g}$ at node $v_{i}$. A link $\ell_{i, j}$ connecting nodes $v_{i}$ and $v_{j}$ is added to the graph if the Euclidean distance between the nodes is less than $\Re$, with all nodes distributed randomly. The FRMA and BRMA packets can be deployed to find the optimal path between a node $\mathrm{i}$ and active node $\mathrm{j}$. Each link $\ell_{i, j}$ connecting nodes $v_{i}$ and $v_{j}$ is associated with pheromone $\varphi_{i, j}$.

Route maintenance: The second phase of the proposed routing algorithm called route maintenance, which is responsible for the improvement of routes during the communication. The proposed AODV_ALMA need special packets for route maintenance in MANET communication called Hello Messages. The route maintenance in gateway is done by data packets forwarded by active nodes. The FRMA and BRMA packets establish the pheromone tracks from the source to the gateway nodes. The established paths do not keep their initial pheromone values forever; however it varies based on the data packets traversed with respect to a predefined value.

When a node $v_{i}$ relays a data packet toward the gateway $v_{g}$ to a neighbor node $v_{j}$, it increases the pheromone value of the entry $\left(v_{g}, v_{j}, \varphi\right)$ by $\Delta \varphi$, i.e., the path to the gateway is strengthened by the data packets. In contrast, the next hop $v_{j}$ increases the pheromone value of entry $\left(v_{s}, v_{i}, \varphi\right)$ by $\Delta \varphi$, i.e., the path to the source is also strengthened. The evaporation process of the real pheromone is simulated by regular decreasing of the pheromone value according to the equations (3) given below.

$$
\varphi_{i, j}:=(1-q) \cdot \varphi_{i, j} \quad q \in[0,1]
$$


Mobile nodes recognize duplicate reception of data packets, based on the source address and sequence number in IPv6 routing header. If a mobile node receives a duplicate packet, it sets the DUPLICATE_ERROR flag and sends the packet back to the previous node. The previous node deactivates the link to this node, so that data packets are not sent in this direction any more.

Route failure handling: The third phase of proposed algorithm handles routing failures, which are caused especially through active mobile node mobility and the change of gateway access by the source mobile node in hybrid MANET. The proposed scheme recognizes the route failure through a missing acknowledgment from MAC layer. If the mobile active node in the path of the gateway is moving it sends the FRMA packet based on the source address available in the routing table. The FRMA packet generated will handle the route failure transparency to the source and the active nodes without loss of data packets by maintaining high network connectivity towards the gateway. If a mobile node gets a ROUTE_ERROR message for a certain link, it first deactivates this link by setting the hop count to 0 and also the pheromone value to 0 .

The mobile node searches for an alternate link or gateway in its routing table. If a second gateway link with the pheromone decay time not reaching the minimum threshold value available, it sends the data packet via this path. Otherwise the node informs its neighbor active nodes, hoping that they can relay the packet.

Performance evalutaion : The performance evaluation of proposed algorithm is compared with existing proposals Wakikwa and Jelger: the network simulator ns-2 is used. The source code of AODV modified with the proposal of Wakikwa called as AODV_Wakikwa and the code of OLSR modified with the proposal of Jelger called as OLSR_Jelger. The source code for the AODV_ALMA written using the Mobile Agents supplied as open source.

\begin{tabular}{ll} 
Simulation Scenario & \\
\hline Transmission Range & $250 \mathrm{mts}$ \\
Physical Layer & Two-ray propagation \\
Link Layer & IEEE 802.11 \\
Queue Buffer size & 64 packets \\
Routing buffer size & 50 packets \\
Channel Capacity & 2 Mbps \\
No. of gateway & 3 \\
No. of mobile node & 50 \\
Area & $1500 \times 300 \mathrm{mts}$ \\
Speed & $0-10 \mathrm{~m} / \mathrm{s}$ \\
Pause time & $0-600 \mathrm{sec}$ \\
Traffic type & CBR \\
Sources & 10 \\
Sending rate & 4 packets/sec \\
Packet size & 512 bytes \\
Mobile agent packet & 10 \\
History Size & 6 hops \\
Simulation Time & 900 seconds \\
\hline
\end{tabular}

\section{RESULTS}

The main performance metrics under consideration are those recommended in RFC 2501:

* Packet delivery Ratio (PDR): the percentage of successfully delivered data packets over all the data packets sent out by the sources.

* Normalized control overhead: the total number of control packets sent and forwarded over the number of successfully delivered data packets. This metric represents the cost in terms of overhead of the different approaches.

* Gateway discovery overhead: the total number of control messages associated with the discovery of gateways. This metric provides information regarding how much of the control packet overhead is due to the Internet connectivity scheme.

* Connectivity: the average number of mobile nodes in the hybrid MANET for which a node has unexpired routes.

* End-to-End delay: the time taken to send the data from source to the destination normalized with the time taken to discover the route.

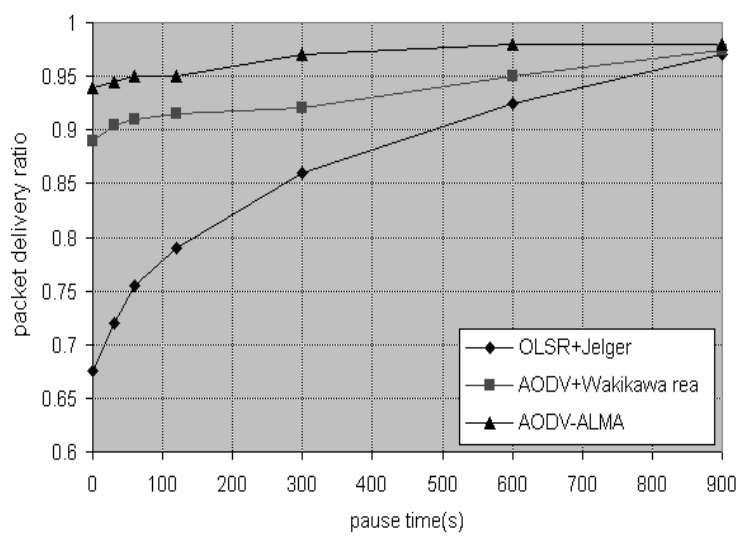

Fig. 3: Packet delivery ratio

Packet delivery ratio (PDR): The simulation comparisons for AODV_ALMA, AODV_Wakikawa and OLSR_Jelger are shown in Fig. 3. The PDR of AODV_ALMA is high as that of reactive AODV_Wakikawa. The change in performance for the highest pause times caused by the fact that the network is quite sparse and in the same time quite static: this means that nodes can become unreachable for long periods. The OLSR_Jelger is having very less PDR compared with AODV_ALMA; the reason is that link break in the path either to the gateway or in MANET is handled after some time gap. In AODV_ALMA the link break to the gateway is monitored by change in the pheromone value. The pheromone decay time is associated with data packets transmitted to Internet host in the established track by the ALMA packets. After carefully analyzing the simulations, AODV_ALMA using the pheromone value to adaptively select next hop 


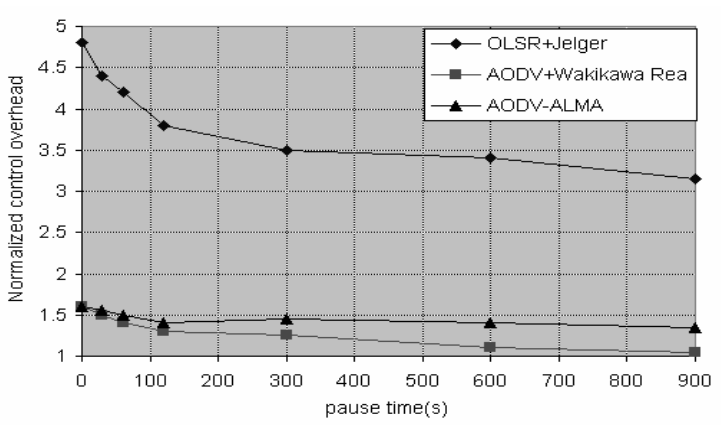

Fig. 4: Normalizedcontroloverhead

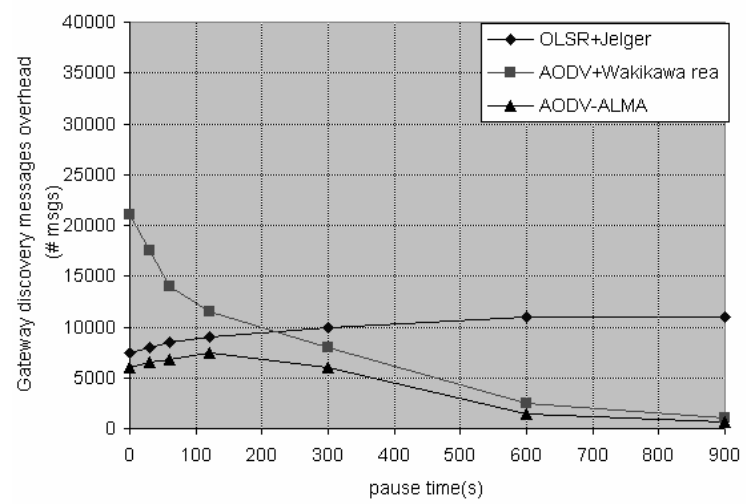

Fig. 5: Gateway discovery overhead

to gateway makes the topology changes strong to mobility.

Normalized control overhead: The overhead comparison for AODV_ALMA, AODV_Wakikawa and OLSR_Jelger is shown in Fig. 4. In OLSR_Jelger, due to its proactive nature has high control overhead for high mobility conditions. As the mobility becomes a static overhead decrease but not as less than AODV_Wakikawa. In AODV_Wakikawa, due to its reactive nature has less control overhead for high mobility conditions. It is reducing further as the network becomes static. In AODV_ALMA, control overhead is optimized to that of AODV_Wakikawa in high mobility conditions. It is slightly higher for low mobility conditions. but becomes slightly higher in static conditions.

Gateway discovery overhead: The gateway discovery function is different in all the proposals, is shown in Fig. 5. In AODV_ALMA gateway discovery overhead is less for high and low mobility conditions. This is due to the size of and number of mobile agent packets generated in a multicast manner with priority in transmission. In AODV_Wakikawa the gateway discovery overhead is higher in high mobility conditions. This is due to the fact time values (TTL) is set to discover the gateway and no change for mobility conditions. In OLSR_Jelger due to its limited periodic flooding, gateway discovery overhead is higher

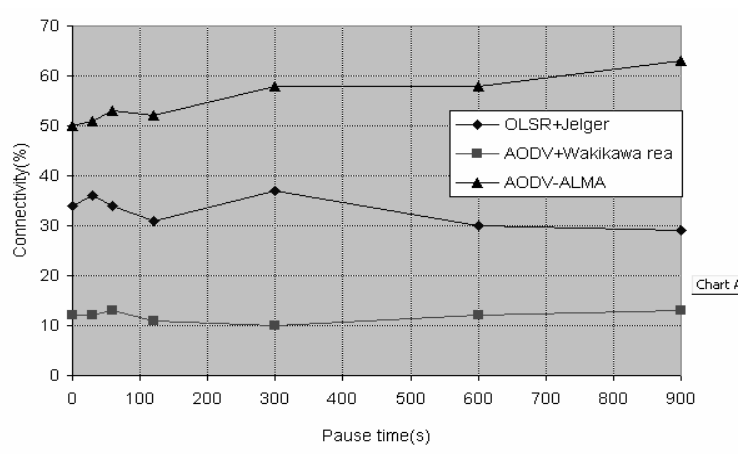

Fig. 6: Connectivity maintenance

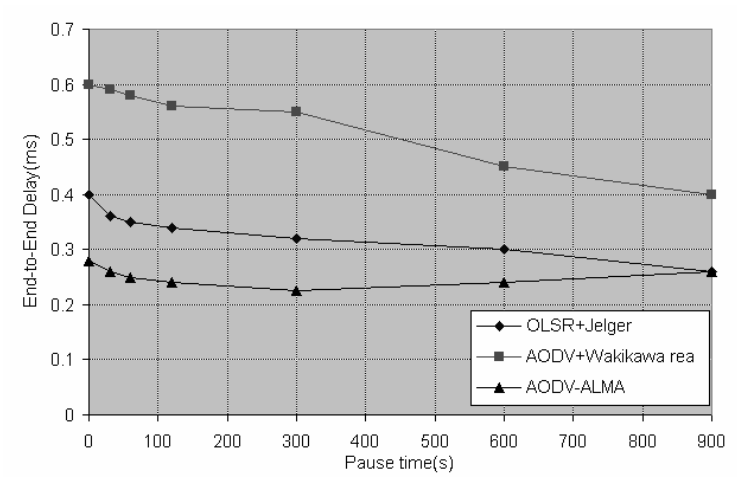

Fig. 7: End-to-end delay

compared to that of AODV_ALAM and varies with mobility.

Connectivity maintenance: The average number of nodes having unexpired route to the gateway is used as a measure for connectivity is shown in Fig. 6. In AODV_ALMA connectivity is high for high mobility conditions. This is due to its adaptive pheromone value changes and updating by data packets forwarded to the gateway. The connectivity is tracked by mobile agents to select the new active nodes for forwarding. In OLSR_Jelger connectivity is slightly lesser than AODV_ALMA with proactive route updating. In AODV_Wakikawa connectivity is very less due to the rediscovery of new routes to the gateway and to changes in link break for high mobility conditions.

End-to-end delay: The end-to-end delay includes buffering, queuing delay at interface queue, retransmission delay, propagation and transfer time delay. The assumption is that all other delay values are same except the route discovery delay. As shown in Fig. 7, AODV_ALMA is having less delay due to separate route discovery process for the gateway and for the local MANET In different mobility conditions the end-to-end delay is variable, due to changes in pheromone value in particular link. In OLSR_Jelger proactive nature of route discovery has slightly higher delay due to the time gap in finding the route. In AODV_Wakikawa reactive nature of route discovery first locally then globally has higher delay. The time 
extension value (TTL) for initial route discovery in MANET up to network diameter and after some time for the gateway vary with mobility conditions.

\section{CONCLUSION}

The paper proposed a new adaptive gateway discovery mechanism AODV_ALMA based on hybrid mobile agent approach. The performance tradeoffs and limitations with upcoming proposals are compared using simulation. The proposed approach increased the network connectivity of mobile nodes with gateway and reduced the end-to-end delay for packet transmission to Internet. The results tested for mobility condition only, it can also be tested for different traffic conditions and increased scalability. In the near further other internetworking related issues such as improved DAD mechanism, efficient support of DNS, discovery of application and network services, network authentication and integrated security mechanism have to be enhanced with the proposed mechanism.

\section{REFERENCES}

1. Scott, M.C., J.P. Macket and G.H. Cirincione, 1999. Internet based mobile ad hoc networking. IEEE Internet Computing, pp : 63-70.

2. Matsuo, H. and K. Mori, 2001. Accelerated ants routing in dynamic networks. Proc. Intl. Conf. on Software Engineering, Artificial Intelligence, Networking and Parallel/Distributed Computing, pp: 333-339.

3. Choudhary, R.R., S. Bhandhopadhyay and K. Paul, 2000. A distributed mechanism for topology discovery in ad hoc wireless networks using mobile agents. Proc. Mobicom, pp: 145-146.
4. Broch, J., D.A. Maltz and D.B. Johnson, 1999. Supporting hierarchy and heterogeneous interfaces in multi-hop wireless ad hoc networks. Proc. Workshop on Mobile Computing, Perth, Australia, pp: 85-97.

5. Lei, H. and C. Perkins, 1997. Ad hoc networking with mobile IP. Proc. Second European Personal Mobile Communications Conf., Bonn, Germany, pp: 294-309.

6. Ammari, H. and H.El-Rewini, 2004. Performance evaluation of hybrid environments with mobile gateways. Proc. 9th Int'l Symp Comp. and Comm., 1: 152-57.

7. Ratanchandani, P. and R. Kravets, 2003. A hybrid approach to internet connectivity for mobile ad hoc networks. Proc. IEEE Wireless Communications and Networking Conf., 1: 295-304.

8. Lee, J., 2003. Hybrid gateway advertisement scheme for connecting mobile ad hoc networks to the internet. Proc. 57th IEEE VTC, 1: 191-95.

9. Ruiz, P.-M. and A.-F Gomez-Skarmeta, 2005. Adaptive gateway discovery mechanisms to enhance internet connectivity for mobile ad hoc networks. Ad Hoc and Sensor Wireless Networks, 1: 159-77.

10. Wakikawa, R., J. Malinen, C. Perkins, A. Nilsson and A. Tuominen, 2003. Global connectivity for IPv6 mobile ad hoc networks. (work in progress), IETF Internet Draft, draft-wakikawa-manetglobalv6-03.txt.

11. Jelger, C., T. Noel and A. Frey, 2004. Gateway and address auto configuration for IPv6 ad hoc networks. Internet-draft, draft-jelger-manetgateway-autoconf-v6-02.txt.

12. Singh, S. et al., 2005. Ad hoc network auto configuration: Definition and problem statement, Internet draft. draft-singh-autoconf-adp-01.txt. 